\title{
BAYES CLASS AND WALD CLASS
}

\section{HIROKICHI KUDŌ}

Dedicated to Professor K. Noshiro on the occation of his 60 th birthday

\$1. Introduction and motivation. In this paper we shall study relations among the class $\boldsymbol{B}$ of the Bayes solutions in the strict sense, the class $\boldsymbol{W}$ of the Bayes solutions in the wide sense and the closure $\mathfrak{c}(\boldsymbol{B})$, in a certain sense, of the class $\boldsymbol{B}$. For abbreviation, we shall use the word "Bayes class" for the class $\boldsymbol{B}$ and "Wald class" for the class $\boldsymbol{W}$.

The concepts of Bayes class and Wald class are introduced in connection with the concept of complete class of statistical decision functions, and play important roles in the theory of statistical decision functions. According to Wald-LeCam theorem ([6], [4]), the closure $\mathfrak{c}(\boldsymbol{B})$ of the Bayes class, the Wald class $\boldsymbol{W}$ and the intersection of $\mathfrak{c}(\boldsymbol{B})$ and $\boldsymbol{W}$ are complete classes of decision functions under certain conditions. Many results have been published on the problem of constructing complete classes. As far as the author knows, these results are proved on the basis of the completeness of the class $\mathfrak{c}(\boldsymbol{B})$, but neither of $\boldsymbol{W}$ nor $\boldsymbol{W} \cap \mathfrak{c}(\boldsymbol{B})$. From this fact, we may expect a possibility that in most regular cases $\mathfrak{c}(\boldsymbol{B})$ will be contained in $\boldsymbol{W}$. Theorems we shall give in this paper show that our expectation is quite reasonable (see Thms. 4,6,9,11,12, and 15). Of course, this is not always the case. Recently T. Kusama [3] gave an example in which $\mathfrak{c}(\boldsymbol{B})$ coincides with the whole space of decision functions.

Another question arises along this line. Wald proved in his book [6], under his Assumption 3.7, that both of $\mathfrak{c}(\boldsymbol{B})$ and $\boldsymbol{W}$ coincide with the Bayes class $\boldsymbol{B}$ itself (Theorem 3.15 and 3.16 in [6]) . Assumption 3.7 in [6] is essentially that the parameter space $T$ is compact in some sense and the loss function is equicontinuous as a family of functions on $T$. These facts suggest us another possibility that the assumptions of the compactness of the parameter space and the equicontinuity of the loss function will imply important conclusions (e.g., see Thms. 14 and 15).

Received July 27, 1966. 
The above two possibilities motivated the present research. The third motivation occurs from the results in [2] and [5]. The author proved in [2] that in certain problems of testing hypotheses $\mathfrak{c}(\boldsymbol{B})$ is contained in the class of decision functions which minimize the value of a distribution (in the sense of $L$. Schwarz: Théorie des distributions) on the space of risk functions. On the other hand, J. Sacks [5] showed that in estimation problems $\mathfrak{c}(\boldsymbol{B})$ is contained in the class of decision functions which minimize, in a certain sense, the value of the integral of risk function with respect to a $\sigma$-finite measure though the integral is not finite. These results suggest us that, under regular conditions, the closure $\mathfrak{c}(\boldsymbol{B})$ of the Bayes class will be contained in the class of "generalized Bayes solutions" relative to a linear functional which is a limit, in a certain sense, of prior probability measures. If this is the case, it becomes reasonably handy to construct a nontrivial complete class of decision functions, because the complet class will be given as the class of all the generalized Bayes solutions obtained by the method of minimum posterior loss.

In Section 2 we shall discuss the Bayes class and the Wald class in an abstract set $X$ relative to a family $F$ of functions on $X$ for the purpose of clarifying the point of arguments, and topological discussions of function spaces are the main tool. In Section 3 we shall consider $X$ and $F$ as a class of functions and a class of measures, respectively, on a topological space. We shall not specify the form of elements in the class $X$, but the reader may regard $X$ as the class $\{r(\cdot, \delta): \delta \in D\}$ of the risk functions with the space $D$ of decision functions $\delta$. Each one of the theorems in these sections contains a relation or relations between the Bayes class and the Wald class under the respective condition. Particularly, Theorems 5, 6, 9, 14 and 15 give the relation expected in the first two motivations. In Section 4 we shall sketch how to approach the third problem, but no precise discription will be given for this approach.

§2. Bayes class and Wald class relative to a class of functions. Let $X$ be an arbitrary set and $F$ a class of real-valued non-negative functions of $x \in X$.

Definition 1. An element $x$ in $X$ is called a Bayes point relative to a function $f$ in $F$, if $x$ satisfies

$$
f(x)=\min _{y \in X} f(y) .
$$


If $x$ is a Bayes point relative to an element of $F$, we shall simply call it a Bayes point. The set $\boldsymbol{B}[F]$ of all Bayes points is called the Bayes class relative to $F$, or simply the Bayes class.

Definition 2. The set $\boldsymbol{W}[F]$ of all points $x \in X$ which satisfy

$$
\inf _{f \in F}\left\{f(x)-\inf _{y \in X} f(y)\right\}=0
$$

is called the Wald class relative to $F$. An element $x$ of $W[F]$ is called a Wald point.

In the following discussions we shall treat of relations among the Bayes class, the Wald class and their closures in the sense of a certain topology in $X$ which will be introduced afterward. Though we shall regard, in Section $3, X$ as a class of functions on a certain space $T$ and $F$ as a class of measures on $T$, we do not confine them in any aspect in the present section.

Let $\subseteq$ be a non-void family of subsets of $X$, and denote by $\mathscr{T}_{\mathfrak{S}}$ the topology induced on the space of real-valued nonnegative functions on $X$ by the system of neighborhoods of a real-valued function $f$ :

$$
V_{\Im}(f: S, \varepsilon)=\left\{g: \sup _{x \in S}|f(x)-g(x)|<\varepsilon\right\},
$$

with an element $S$ of $\mathbb{S}$ and a positive number $\varepsilon$. By $(F, \mathbb{S})$ we mean the space $F$ equipped with the relative topology induced by $\mathscr{T}_{\mathcal{S}}$, and we use the notation " $\mathscr{T}_{\mathscr{S}}$." for the phrase "in the sense of $\mathscr{T}_{\mathscr{S}}$ ". The $\mathscr{T}_{\mathbb{S}}$-closure of $F$ will be denoted by $\subseteq(F)$. The family consisting only of the single element $X$ is denoted by $\mathfrak{t}$, and the family of all finite subsets of $X$ by $\mathfrak{w}$. In this paper we shall use these notations $\mathfrak{t}$ and $\mathfrak{w}$ in the place of $\mathfrak{S}$; for instance $\mathfrak{t}(F)$ will stand for the closure of $F$ in the uniform convergence topology $\mathscr{T}_{\mathfrak{u}}$, and " $\mathscr{T}_{\mathfrak{w}}$-compact" means "compact in the pointwise convergence topology $\mathscr{T}_{\mathfrak{w}}$ in the function space".

$$
\begin{array}{lll}
\text { Theorem 1. } & \text { (i) } & \boldsymbol{B}[F] \subset \boldsymbol{W}[F], \\
& \text { (ii) } & \boldsymbol{B}[\mathfrak{u}(F)] \subset \boldsymbol{B}[\mathfrak{w}(F)], \\
& \text { (iii) } & \boldsymbol{B}[\mathfrak{u}(F)] \subset \boldsymbol{W}[F] .
\end{array}
$$

Proof. The statements (i) and (ii) are evident. To prove the statement (iii), let $x$ be in $B[\mathfrak{t}(F)]$. Then there is a function $f$ in $\mathfrak{t}(F)$ such that

$$
f(x)=\min _{y \in X} f(y) .
$$


Since $f \in \mathfrak{t}(F)$, for any $\varepsilon>0$ there is a function $g \in F$ such that $\mid f(y)-$ $g(y) \mid<\varepsilon$ for every $y \in X$. Let $z$ be a point of $X$ for which $g(z)<\inf _{y \in X} g(y)+\varepsilon$. Then we have $g(x)-\inf _{y \in X} g(y)<g(x)-g(z)+\varepsilon=(g(x)$ $-f(x))+(f(x)-f(z))+(f(z)-g(z))+\varepsilon<3 \varepsilon$, which means that

$$
\inf _{g \in F}\left\{g(x)-\inf _{y \in X} g(y)\right\}<3 \varepsilon
$$

Since $\varepsilon$ is arbitrary, we have $x \in \boldsymbol{W}[F]$.

THEOREM 2. Suppose that for any pair $\{x, y\}$ of points of $X$ there is an element $S$ of $\subseteq$ such that $x$ and $y$ belong to $S$. If $F$ is relatively $\mathscr{T}_{\mathbb{S}}$-compact, then we have

$$
\boldsymbol{W}[F] \subset \boldsymbol{B}[\Im(F)]=\boldsymbol{W}[\Im(F)] .
$$

Proof. Let $x \in \boldsymbol{W}[F]$. Then there is a sequence $\left\{f_{n}\right\}$ of functions in $F$ such that

$$
\lim _{n \rightarrow \infty}\left\{f_{n}(x)-\inf _{y \in X} f_{n}(y)\right\}=0,
$$

that is to say, for any $\varepsilon>0$ there is a positive integer $n_{0}$ such that for any $n$ larger than $n_{0}$

$$
f_{n}(x)-\inf _{y \in X} f_{n}(y)<\varepsilon
$$

Since $\mathfrak{S}(F)$ is $\mathscr{T}_{\mathfrak{S}}$-compact, there is a $\mathscr{T}_{\mathfrak{S}}$-cluster points $f_{0}$ in $\mathfrak{S}(F)$ of the sequence $\left\{f_{n}\right\}$. Take a point $z$ in $X$ for which $f_{0}(z)<\inf _{y \in X} f_{0}(y)+\varepsilon$. By the assumption there exists an element $S$ of $\subseteq$ such that $x$ and $z$ belong to $S$, and by the definition of $f_{0}$ there exists a positive integer $n>n_{0}$ such that $\left|f_{n}(y)-f_{0}(y)\right|<\varepsilon$ for every $y$ in $S$. For such an $n$ we have

$$
\begin{aligned}
f_{0}(x)-\inf _{y \in X} f_{0}(y)<f_{0}(x)-f_{0}(z)+ & \varepsilon<f_{n}(x)-f_{n}(z)+3 \varepsilon \\
& <f_{n}(x)-\inf _{y \in X} f_{n}(y)+3 \varepsilon<4 \varepsilon .
\end{aligned}
$$

Since $\varepsilon$ is arbitrary, we have $f_{0}(x)=\min _{y \in X} f_{0}(y)$, or $x \in \boldsymbol{B}[\Im(F)]$. Thus we have $\boldsymbol{W}[F] \subset \boldsymbol{B}\left[\varsigma_{(F)]}\right.$. Replacing $F$ by $\mathfrak{S}(F)$ in the formula just shown, 
we have $\boldsymbol{W}[\Im(F)] \subset \boldsymbol{B}[\subseteq(F)]$. Combining with the statement (i) of Thoerem 1 , we get the desired result.

Remark. The assumption on $\mathfrak{S}$ in Theorem 2 holds true for $\mathfrak{S}=\mathfrak{t}$ and $\mathfrak{w}$, so that the relation (1) holds in both cases. Particularly, if $\mathfrak{n}(F)$ is $\mathscr{T}_{\mathfrak{u}}$-compact, then

$$
\boldsymbol{B}[\mathfrak{i}(F)]=\boldsymbol{W}[F]=\boldsymbol{W}[\mathfrak{u}(F)] .
$$

Theorem 3. If $\mathfrak{H}(F)$ is $\mathscr{T}_{\mathfrak{w}}$-compact, then

$$
\boldsymbol{W}[F]=\boldsymbol{B}[\mathfrak{u}(F)]=\boldsymbol{B}[\mathfrak{w}(F)]=\boldsymbol{W}[\mathfrak{u}(F)]=\boldsymbol{W}[\mathfrak{w}(F)] .
$$

Proof. Since $(-\infty,+\infty)^{X}$ is a Hausdorff space in $\mathscr{T}_{\mathfrak{w}}$-sense and $\mathfrak{t}(F)$ is $\mathscr{T}_{\mathfrak{w}}$-compact, it is $\mathscr{T}_{\mathfrak{w}}$-closed, and so $\mathfrak{H}(F)=\mathfrak{w}(F)$. From this it follows directly that $\boldsymbol{B}[\mathfrak{u}(F)]=\boldsymbol{B}[\mathfrak{w}(F)]$ and $\boldsymbol{W}[\mathfrak{u}(F)]=\boldsymbol{W}[\mathfrak{w}(F)]$. Since it is easy to verify that $\mathfrak{w}(F)$ is $\mathscr{T}_{\mathfrak{w}}$-compact, we have $\boldsymbol{W}[F] \subset \boldsymbol{B}[\mathfrak{w}(F)]=\boldsymbol{W}[\mathfrak{w}(F)]$ by Theorem 2 and Remark. On the other hand, it follows from (iii) of Theorem 1 that $\boldsymbol{B}[\mathfrak{t}(F)] \subset \boldsymbol{W}[F]$. Thus, combining these relations, we have our desired formula (2).

In the sequel of this section, we shall assume that $X$ is a topological space, and denote the closure of a subset $Y$ of $X$ by $\mathfrak{c}(Y)$. Let $\mathfrak{i}$ be the collection of all compact subsets of $X$. When we take this instead of $\subseteq$, the topology $\mathscr{T}_{\mathfrak{f}}$ can be defined in $F$ in the same way as $\mathscr{T}_{\mathscr{G}}$ be done, and $\mathfrak{f}(F)$ will be used for the $\mathscr{T}_{\mathfrak{k}}$-closure of $F$. The assumption on $\mathfrak{S}$ in Theorem 2 is obviously satisfied by $\mathfrak{S}=\mathfrak{t}$. From Theorems 1,2 and 3 we have:

$$
\begin{aligned}
& \text { 1) } \boldsymbol{B}[\mathfrak{u}(F)] \subset \boldsymbol{B}[\mathfrak{f}(F)] \subset \boldsymbol{B}[\mathfrak{w}(F)], \\
& \text { 2) If } F \text { is relatively } \mathscr{T}_{\mathfrak{f}} \text {-compact, then } \\
& \boldsymbol{W}[F] \subset \boldsymbol{B}[\mathfrak{t}(F)]=\boldsymbol{W}[\mathfrak{f}(F)] . \\
& \text { 3) If } \mathfrak{u}(F) \text { is } \mathscr{T}_{\mathfrak{w}} \text {-compact, then } \\
& \boldsymbol{W}[F]=\boldsymbol{B}[\mathfrak{f}(F)]=\boldsymbol{W}[\mathfrak{f}(F)]=\boldsymbol{B}[\mathfrak{w}(F)]=\boldsymbol{W}[\mathfrak{w}(F)]=\boldsymbol{B}[\mathfrak{t}(F) \mathfrak{j}=\boldsymbol{W}[\mathfrak{u}(F)] .
\end{aligned}
$$

Theorem 4. Suppose that $X$ be a topological space and $F$ a family of lower semi-continuous functions on $X . \quad$ If $\mathfrak{u}(F)$ is $\mathscr{T}_{\mathfrak{u}}$-compact, then

$$
\mathfrak{c}(\boldsymbol{B}[F]) \subset \boldsymbol{B}[\mathfrak{u}(F)]=\mathfrak{c}(\boldsymbol{B}[\mathfrak{u}(F)])=\boldsymbol{W}[F]=\boldsymbol{W}[\mathfrak{u}(F)] .
$$

Proof. Let $x \in \mathfrak{c}(\boldsymbol{B}[F])$. Then for any neighborhood $U$ of $x$ there 
exists a point $y \in \boldsymbol{B}[F] \cap U$. For such a $y$ there corresponds a function $f_{y}$ in $F$ such that $f_{y}(y)=\inf _{z \in X} f_{y}(z)$. Since $\mathfrak{u}(F)$ is $\mathscr{T}_{\mathfrak{u}}$-compact, there is a function $f_{0}$ in $\mathfrak{u}(F)$ which belongs commonly to the $\mathscr{T}_{\mathfrak{u}}$-closure of $\left\{f_{y}: y \in \boldsymbol{B}[F] \cap U\right\}$ for every neighborhood $U$ of $x$. Since the uniform limit function $f_{0}$ of lower semi-continuous functions is also lower semicontinuous, for any $\varepsilon>0$ there is a neighborhood $U_{0}$ of $x$ such that $f_{0}(y)>f_{0}(x)-\varepsilon$ holds for every $y \in U_{0}$. Take a point $y \in U_{0} \cap \boldsymbol{B}[F]$ for which $\left|f_{0}(z)-f_{y}(z)\right|<\varepsilon$ holds for every $z \in X$. Let $z$ be a generic element in $X$. Then $f_{0}(x)-f_{0}(z)=\left(f_{0}(x)-f_{0}(y)\right)+\left(f_{0}(y)-f_{y}(y)\right)$ $+\left(f_{y}(y)-f_{y}(z)\right)+\left(f_{y}(z)-f_{0}(z)\right)<3 \varepsilon$. Since $\varepsilon$ is arbitrary, it holds that $f_{0}(x) \leqq f_{0}(z)$ for all $z \in X$ and that $x \in \boldsymbol{B}[\mathfrak{u}(F)]$, which means $\mathfrak{c}(\boldsymbol{B}[F]) \subset \boldsymbol{B}[\mathfrak{u}(F)]$. Replacing $F$ by $\mathfrak{u}(F)$ in the last formula, we have $\mathfrak{c}(\boldsymbol{B}[\mathfrak{u}(F)]) \subset \boldsymbol{B}[\mathfrak{u}(F)]$. Since the other direction of inclusion is trivial, we get $\boldsymbol{B}[\mathfrak{u}(F)]=\mathfrak{c}(\boldsymbol{B}[\mathfrak{u}(F)])$. The remainders of (3) are due to Remark preceding to Theorem 3.

THeORem 5. Suppose that $X$ be a locally compact Hausdorff space and $F$ be a family of lower semi-continuous functions on $X$. If $\mathfrak{f}(F)$ is $\mathscr{T}_{\mathfrak{t}}$-compact, then

(i) $\quad \mathfrak{c}(\boldsymbol{B}[F]) \subset \boldsymbol{B}[\mathfrak{f}(F)]=\mathfrak{c}(\boldsymbol{B}[\mathfrak{f}(F)])$,

(ii) $\boldsymbol{W}[F] \subset \boldsymbol{B}[\mathfrak{f}(F)]=\boldsymbol{W}[\mathfrak{t}(F)]$.

Proof. Let $x \in \mathfrak{c}(\boldsymbol{B}[F])$. For any neighborhood $U$ of $x$ there is a point $y \in U \cap \boldsymbol{B}[F]$, which corresponds to a function $f_{y} \in F$ such that $f_{y}(y)=$ $\min _{z \in X} f_{y}(z)$. From the $\mathscr{T}_{\mathfrak{t}}$-compactness of $\mathfrak{t}(F)$, there exists a common function $f_{0}$ in $\mathfrak{l}(F)$ which belongs to every sets $\mathfrak{f}\left(\left\{f_{y}: y \in U \cap \boldsymbol{B}[F]\right\}\right)$. Since every $\mathscr{T}_{\mathfrak{t}}$-limit functions of lower semi-continuous functions are also lower semi-continuous, for any $\varepsilon>0$ there is a compact neighborhood $U_{0}$ of $x$ such that $f_{0}(y)>f_{0}(x)-\varepsilon$ for every $y \in U_{0}$. Let $z$ be a generic point of $X$, and then put $E=U_{0} \cup\{z\}$. Then there is a function $f_{y}$ in $V_{\sharp}\left(f_{0}: E, \varepsilon\right)$ for some $y \in U_{0} \cap \boldsymbol{B}[F]$. Therefore we have $f_{0}(x)-f_{0}(z)=\left(f_{0}(x)-f_{0}(y)\right)+$ $\left(f_{0}(y)-f_{y}(y)\right)+\left(f_{y}(y)-f_{y}(z)\right)+\left(f_{y}(z)-f_{0}(z)\right)<3 \varepsilon$. Since $\varepsilon$ is arbitrary, we have $f_{0}(x)=\min _{z \in X} f_{0}(z)$, or $x \in \boldsymbol{B}[\mathfrak{t}(F)]$. Thus we have $\mathfrak{c}(\boldsymbol{B}[F]) \subset \boldsymbol{B}[\mathfrak{t}(F)]$. The other part of (i) is a direct implication of the fact just proved. The statement (ii) is nothing but the result of Theorem 2 in a special case $\subseteq=\mathfrak{~}$

Theorem 6. Suppose that $X$ be a locally compact Hausdorff space and $F$ be 
an equicontinuous family of functions on $X . \quad$ If $\sup _{f \in F} f(x)<\infty$ for every $x \in X$, then

$$
\mathfrak{c}(\boldsymbol{B}[F]) \subset \boldsymbol{W}[F] \subset \boldsymbol{B}[\mathfrak{f}(F)]=\mathfrak{c}(\boldsymbol{B}[\mathfrak{f}(F)])=\boldsymbol{W}[\mathfrak{f}(F)] .
$$

Proof. It is sufficient to show that $\mathfrak{c}(\boldsymbol{B}[F]) \subset \boldsymbol{W}[F]$, because the other relations are direct implications of Theorem 5 and Ascoli theorem $[1, \mathrm{Ch}$. $X]$. Let $x \in \mathfrak{c}(\boldsymbol{B}[F])$ and $\varepsilon$ be a given positive number. From the equicontinuity of $F$ and consequently of $\mathfrak{f}(F)$, there is a compact neighborhood $U_{0}$ of $x$ such that $|f(x)-f(y)|<\varepsilon$ for every $y \in U_{0}$ and every $f \in \mathfrak{H}(F)$. Since $x \in \mathfrak{c}(\boldsymbol{B}[F])$, for any neighborhood $U$ of $x$ there is a point $y \in U \cap \boldsymbol{B}[F]$. Let $f_{y}$ be a function in $F$ such that $f_{y}(y)=\inf _{z \in X} f_{y}(z)$. Since by Ascoli theorem $\mathfrak{f}(F)$ is $\mathscr{T}_{\mathfrak{t}}$-compact, there is a function $f_{0}$ in $\mathfrak{f}(F)$ which is a cluster point $f_{0}$ of the net $\left\{f_{y}\right\}$ in the $\mathscr{T}_{t}$-sense. Take a function $g$ from the intersection of $V_{\mathfrak{f}}\left(f_{0}: U_{0}, \varepsilon\right)$ and $\left\{f_{y} ; y \in U_{0} \cap \boldsymbol{B}[F]\right\}$. Then $g$ has to be $f_{z}$ for some $z \in U_{0} \cap \boldsymbol{B}[F]$, and so $g(x)-g(z)=\left(g(x)-f_{0}(x)\right)+\left(f_{0}(x)-f_{0}(z)\right)+$ $\left(f_{0}(z)-g(z)\right)<3 \varepsilon$. Hence $\inf _{f \in F}\left\{f(x)-\inf _{y \in X} f(y)\right\} \leqq g(x)-g(z)<3 \varepsilon$. Since $\varepsilon$ is arbitrary, $x$ is an element of $\boldsymbol{W}[F]$.

THEOREM 7. Suppose that $X$ be a compact Hausdorff space, and $F$ a class of lower semi-continuous functions on $X$. If for every function $f$ in $\mathfrak{u}(F)$ there is one and only one Bayes point relative to $f$, then we have

$$
\boldsymbol{B}[\mathfrak{u}(F)] \subset \mathfrak{c}(\boldsymbol{B}[F])
$$

Proof. It is sufficient to show that for every $f \in \mathfrak{t}(F)$ there is at least one point $x \in \mathfrak{c}(\boldsymbol{B}[F])$ such that $f(x)=\min _{y \in X} f(y)$. Since $f \in \mathfrak{u}(F)$, for any positive integer $n$ there exists a function $g_{n} \in F$ such that $\left|g_{n}(x)-f(x)\right|<1 / n$ for every $x \in X$. Of course, $g_{n}(x)$ is a lower semi-continuous function and attains its minimum value at a point $x_{n}: g_{n}\left(x_{n}\right)=\min _{y \in X} g_{n}(y)$. Let $x_{0}$ be a cluster point of the sequence $\left\{x_{n}\right\}$, and $\varepsilon$ a given positive number. Then there is a neighborhood $U_{0}$ of $x_{0}$ such that $f\left(x_{0}\right)-\varepsilon<f(y)$ for every $y \in U_{0}$. Let $m$ be a positive integer for which $1 / m<\varepsilon$ and $x_{m} \in U_{0}$. Then for any $y \in X$, we have $f\left(x_{0}\right)-f(y)=\left(f\left(x_{0}\right)-f\left(x_{m}\right)\right)+\left(f\left(x_{m}\right)-g_{m}\left(x_{m}\right)\right)+\left(g_{m}\left(x_{m}\right)-\right.$ $\left.g_{m}(y)\right)+\left(g_{m}(y)-f(y)\right)<3 \varepsilon$, which means that $f\left(x_{0}\right)=\min _{y \in X} f(y)$.

§3. Bayes class and Wald class relative to a class of measures. In this section we shall consider $X$ as a class of bounded continuous non- 
negative functions on a locally compact Hausdorff space $T$, and $F$ as a class of positive and finite Radon measures on $T$, and hence a Bayes point may be called a Bayes function. Denote by $C$ and $C_{0}$ the family of bounded continuous functions on $T$ and the family of continuous functions with compact support on $T$, respectively. Denote by $\Sigma$ the family of all finite positive Radon measures. Since for any $f$ in $\Sigma$ the integral $f(x)=\int x(t) d f$ of $x \in C$ with respect to $f$ can be regarded as a real-valued function defined on $C$, we can introduce, on $\Sigma$, topologies $\mathscr{T}_{\mathfrak{w}}^{C_{0}}, \mathscr{T}_{\mathrm{w}}^{C}$ and etc. Here $\mathscr{T}_{\mathrm{w}}{ }^{C_{0}}$ stands for the topology of $\Sigma$ induced by the system of neighborhoods $V_{\mathfrak{w}}(f: S, \varepsilon)$ with the collection $\mathfrak{w}$ of finite subsets $S$ of $C_{0}$, and $\mathscr{T}_{\mathfrak{w}}{ }^{C}$ is a similar notation to the above for the topology of $\Sigma$ in which $C_{0}$ is replaced by $C$. Besides these topologies we shall consider the so-called norm topology induced by the norm $\|f-g\|=($ total variation of $f-g)$ of $f-g$. As is mentioned at the beginning of this paragraph, we shall define $X$ as a non-void subfamily of $C$ consisting of non-negative functions and $F$ as a non-void subfamily of $\Sigma$ consisting of measures for which there is an $A>0$ such that if $f \in F$ then $\|f\|<A$. In this section we consider several kinds of weak topologies of a function space. To distinguish these topologies, we need to improve our notations by indicating the relevant space, for instance we shall denote by $\mathfrak{w}\left(F ; C_{0}\right)$ the

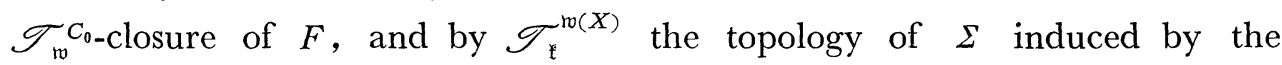
system of neighborhoods $V_{\mathfrak{f}}(f: S, \varepsilon)$ with the collection $\mathfrak{f}$ of $\mathscr{T}_{\mathfrak{w}}$-compact subsets $S$ of the weak closure $\mathfrak{w}(X)$ of $X$. Therefore the notation $\mathfrak{f}(H ; \mathfrak{w}(X))$ stands for the $\mathscr{T}_{t}^{\mathfrak{w}(X)}$-closure of a subset $H$ of $\Sigma$. Though $\mathfrak{w}(X)$ might have to be written as $\mathfrak{w}(X ; T)$, we shall drop this $T$ from the notation, because no confusion will occur.

Before proceeding to our main concerns, we have to give several lemmas.

Lemma 1. It is a necessary and sufficient condition for $\|f\|$ being $\mathscr{T}_{\mathfrak{w}}^{C_{0-}}$ continuous on $\mathfrak{w}\left(F: C_{0}\right)$ that for any $\varepsilon>0$ there is a function $x \in C_{0}$ such that $0 \leqq x(t) \leqq 1$ for every $t \in T$ and

$$
\|f\|-\int x(t) d f<\varepsilon \quad \text { for every } f \in F
$$

In this case $\mathscr{T}_{\mathfrak{w}}^{C}$ coincides with $\mathscr{T}_{\mathfrak{w}}^{C_{0}}$ on $\mathfrak{w}\left(F ; C_{0}\right)$, and hence we have $\mathfrak{w}(F ; C)=$ $\mathfrak{w}\left(F ; C_{0}\right)$. 
The proof is quite similar to that in the case of relatively weakly compact subsets of the set of probability measures, and so it will be omitted here.

Lemma 2. Suppose that the class $X$ of functions is equicontinuous on $T$, and that all the functions in $X$ are bounded by a finite number $B$. If $\|f\|$ is a $\mathscr{T}_{\mathrm{w}}^{C_{0-}}$ continuous function of $f$ on $\mathfrak{w}\left(F ; C_{0}\right)$, then $\mathfrak{w}\left(F ; C_{0}\right)$ is also an equicontinuous family of functions on $\left(\mathfrak{w}(X), \mathscr{T}_{\mathfrak{w}}\right)$, the space $\mathfrak{w}(X)$ equipped with the topology $\mathscr{T}_{\mathfrak{w}}$.

Proof. It follows from Ascoli theorem that $\mathfrak{w}(X)$ is $\mathfrak{f}(X)$. Therefore for any compact subset $E$ of $T$ the $\mathscr{T}_{\mathfrak{\xi}}$-neighbohood $V_{\mathfrak{k}}(x: E, \varepsilon)$ of $x$ in $\mathfrak{w}(X)$ has a common point with $X$. Let $E_{\varepsilon}$ be a compact subset of $T$ such that $\|f\|-f\left(E_{\varepsilon}\right)<\varepsilon$ for every $f \in \mathfrak{w}\left(F: C_{0}\right)$, the existence of which is guaranteed

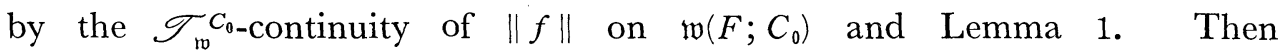
$\left|\int x(t) d f-\int y(t) d f\right|<(A+2 B) \varepsilon$ for every $y \in V_{\mathfrak{t}}\left(x: E_{\varepsilon}, \varepsilon\right) \cap \mathfrak{w}(X)$, which means that $\mathfrak{w}\left(F ; C_{0}\right)$ is an equicontinuous family of functions on $(\mathfrak{w}(X)$, $\left.\mathscr{T}_{\mathfrak{t}}\right)$. Since $\mathscr{T}_{\mathfrak{f}}$ coincides with $\mathscr{T}_{\mathfrak{w}}$ on $\mathfrak{w}(X)$ again by Ascoli theorem, the proof is complete.

Lemma 3. Under the same conditions as in Lemma 2, $\mathscr{T}_{\text {w }}^{C_{0}}$ is a stronger topologv than $\mathscr{T}_{\mathfrak{u}}^{\mathfrak{w}(X)}$, the uniform topology with respect to $\mathfrak{w}(X)$, on the space $\mathfrak{w}\left(F ; C_{0}\right)$.

Proof. From the uniform boundedness of $X$ and Tychonoff theorem it follows that $\mathfrak{w}(X)$ is $\mathscr{T}_{\mathfrak{w}}$-compact. Since $\mathfrak{i w}\left(F ; C_{0}\right)$ is equicontinuous on $\left(\mathfrak{w}(X), \mathscr{T}_{\mathfrak{w}}\right)$ by Lemma 2, it follows from Ascoli theorem that $\mathscr{T}_{\mathfrak{u}}^{\mathfrak{w}(X)}$ coincides with $\mathscr{T}_{\mathfrak{w}}^{\mathfrak{w}(X)}$ on $\mathfrak{w}\left(F ; C_{0}\right)$. Therefore $\mathscr{T}_{\mathfrak{u}}^{\mathfrak{w}(X)}$ is weaker than $\mathscr{T}_{\mathfrak{w}}^{C}$. By the last statement of Lemma 1 , we see that $\mathscr{T}_{\mathfrak{u}}^{\mathfrak{r}(X)}$ is weaker than $\mathscr{T}_{\mathrm{r}} \mathrm{C}_{0}$.

The above two lemmas (Lemma 2 and Lemma 3) are generalized as follows :

Lemma 4. Suppose that for any $x$ in $X$ there corresponds a $\mathscr{T}_{\text {ro }}$-neighborhood $V$ of $x$ such that $V \cap X$ is an equicontinuous family of functions on $T$ and

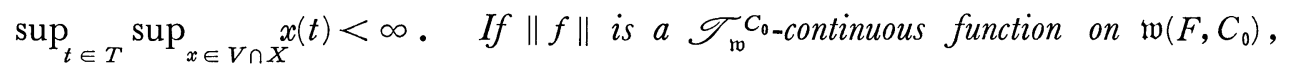
then $\mathfrak{w}\left(F, C_{0}\right)$ is an equicontinuous family of functions on $\left(\mathfrak{w}(X), \mathscr{T}_{\mathfrak{w}}\right)$, and $\mathscr{T}_{\mathfrak{w}}^{C_{0}}$ is stronger than $\mathscr{T}_{\mathfrak{i}}^{\mathfrak{r}(X)}$.

The proof is quite similar to the proofs of Lemmas 2 and 3 .

Theorem 8. Suppose that for any $x$ in $X$ there corresponds a $\mathscr{T}_{\mathfrak{w}}$-neigh- 
borhood $V$ of $x$ such that $V \cap X$ is equicontinuous on $T$ and $\sup _{t \in T} \sup _{y \in V \cap X} y(t)<\infty$. If $\|f\|$ is $\mathscr{T}_{\mathfrak{w}}^{C_{0}-c o n t i n u o u s ~ o n ~} \mathfrak{w}\left(F, C_{0}\right)$, then we have

$$
\begin{aligned}
& \mathfrak{w}(\boldsymbol{B}[F]) \subset \boldsymbol{B}\left[\mathfrak{i w}\left(F ; C_{0}\right)\right]=\mathfrak{w}\left(\boldsymbol{B}\left[\mathfrak{w}\left(F ; C_{0}\right)\right]\right), \text { and } \\
& \boldsymbol{W}[F] \subset \boldsymbol{B}\left[\mathfrak{w}\left(F ; C_{0}\right)\right]=\boldsymbol{W}\left[\mathfrak{w}\left(F ; C_{0}\right)\right] .
\end{aligned}
$$

Proof. Let $x$ be a function in $\mathfrak{w}(\boldsymbol{B}[F])$. Then for any $\mathscr{T}_{\mathfrak{w}}$-neighborhood $V$ of $x$ there is a function $y \in V \cap \boldsymbol{B}[F]$. Let $f_{y}$ be a measure in $F$ such that $\int y(t) d f_{y}=\inf _{z \in X} \int z(t) d f_{y}$. Since the collection of sets $\left\{f_{y}\right.$ : $y \in V \cap \boldsymbol{B}[F]\}$ of measures for every $\mathscr{T}_{w}$-neighborhoods $V$ of $x$ has the finite

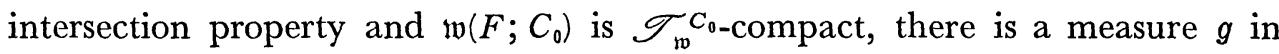
$\mathfrak{w}\left(F ; C_{0}\right) \quad$ which belongs to every $\mathfrak{w}\left(\left\{f_{y}: y \in V \cap \boldsymbol{B}[F]\right\} ; C_{0}\right)$, and so to $\mathfrak{w}\left(\left\{f_{y}: y \in V \cap \boldsymbol{B}[F]\right\} ; \mathfrak{w}(X)\right)$. Since $\mathfrak{i}\left(F ; C_{0}\right)$ is equicontinuous on $\left(\mathfrak{w}(X), \mathscr{T}_{\mathfrak{w}}\right)$ by Lemma 4, it follows from Ascoli theorem that $\mathscr{T}_{\mathfrak{w}}^{\mathfrak{w}(X)}$ is equivalent to $\mathscr{T}_{\mathfrak{t}}^{\mathfrak{w}(X)}$ on $\mathfrak{w}\left(F ; C_{0}\right)$. Therefore $g$ belongs to the $\mathscr{T}_{\mathfrak{t}}^{\mathfrak{w}(X)}$-closure of $\left\{f_{y} ; y \in V \cap \boldsymbol{B}[F]\right\}$. Let $z$ be an arbitrary function in $X$ and $\varepsilon$ an arbitrary positive number. Since $g(x)$ is a continuous function on $\left(X, \mathscr{T}_{\mathfrak{w}}\right)$, there is a $\mathscr{T}_{\mathrm{w}}$-neighborhood $V_{0}$ of $x$ such that $\int y(t) d g>\int x(t) d g-\varepsilon$ for every $y \in V_{0} \cap X$. Take a $\mathscr{T}_{w}$-compact set $E=\mathfrak{w}\left(V_{0}\right) \cap\{z\}$, and consider a $\mathscr{T}_{\mathfrak{t}}{ }^{\mathrm{m}(X)}$-neighborhood $V_{\mathfrak{t}}(g: E, \varepsilon)$ of $g$. Then by a similar calculations to that in the proof of Theorem 5 , we have $\int x(t) d g-\int z(t) d g<3 \varepsilon$. Since $\varepsilon$ is arbitrary, we have $x \in \boldsymbol{B}\left[\mathfrak{w}\left(F ; C_{0}\right)\right]$.

The first half of (5) can be proved in a similar way to Theorem 2 by using $\mathscr{T}_{\mathfrak{w}}{ }^{C}$-compactness of $\mathfrak{w}\left(F ; C_{0}\right)$ instead of the $\mathscr{T}_{\mathfrak{w}}{ }^{X}$-compactness of $\mathfrak{w}(F ; X)$. The rests of our theorem are obvious.

Theorem 9. Suppose that $X$ be an equicontinuous family of functions on $T$ and $\sup _{t \in T} \sup _{x \in X} x(t)<\infty$. If $\|f\|$ is a $\mathscr{T}_{\mathrm{w}}{ }^{C_{0}-\text { continuous function of } f \text { on }}$ $\mathfrak{w}\left(F ; C_{0}\right)$, then we have

$$
\mathfrak{w}(\boldsymbol{B}[F]) \subset \boldsymbol{B}\left[\mathfrak{w}\left(F ; C_{0}\right)\right]=\mathfrak{w}\left(\boldsymbol{B}\left[\mathfrak{w}\left(F ; C_{0}\right)\right]\right)=\boldsymbol{W}[F]=\boldsymbol{W}\left[\mathfrak{w}\left(F ; C_{0}\right)\right] .
$$

Proof. First we get the relations (4) and (5) as a special case of Theorem 8. Since $\mathfrak{w}(X)$ is $\mathscr{T}_{\mathfrak{w}}$-compact by Tychonoff theorem, the equicontinuous family $F$ of functions on $\mathfrak{w}(X)$ has a $\mathscr{T}_{\mathfrak{w}}^{\mathfrak{w}(X)}$-closure $\mathfrak{w}(F ; \mathfrak{w}(X))$ which is $\mathscr{T}_{\mathfrak{u}}^{\mathfrak{w}(X)}$-compact and coincides with $\mathfrak{u}(F ; \mathfrak{w}(X))$. Therefore, from Remark 
preceding to Theorem 3, it follows that $\boldsymbol{W}[F]=\boldsymbol{W}[\mathfrak{w}(F ; \mathfrak{w}(X))]$. Combining with a trivial relation $F \subset \mathfrak{w}(F ; C) \subset \mathfrak{w}(F ; \mathfrak{w}(X))$ and Lemma 1 , we have our desired result.

Theorem 10. Let $X$ satisfy the same conditions as in Theorem 9, and $G$ be $a \mathscr{T}_{\mathfrak{w}}^{C_{0}-d e n s e}$ subset of $F$. Suppose that for any measure $f$ in $F$ there corresponds a subset $K_{f}$ of $G$ such that $f \in \mathfrak{w}\left(K_{f} ; C_{0}\right) \subset F$. If $\|f\|$ is a $\mathscr{T}_{\mathfrak{w}}^{C_{0} \text {-continuous }}$ function of $f$ on $F$, then we have

$$
\boldsymbol{B}[F] \subset \boldsymbol{W}[G] .
$$

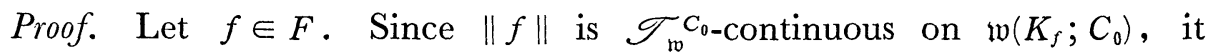
follows from Theorem 9 that $\boldsymbol{B}\left[\mathfrak{t}\left(K_{f} ; C_{0}\right)\right]=\boldsymbol{W}\left[K_{f}\right] \subset \boldsymbol{W}[G]$, and hence any Bayes function in $X$ relative to $f$ belongs to $\boldsymbol{W}[G]$. Thus we have our desired result.

Theorem 11. Suppose that $X, F$ and $G$ satisfy the same conditions as in Theorem 10. If for every measure $f$ in $F$ the Bayes function relative to $f$ is uniquely determined and if $X$ is $\mathscr{T}_{\mathrm{w}}$-closed in $C$, then we have

$$
\boldsymbol{B}[F] \subset \mathfrak{w}(\boldsymbol{B}[G]) \subset \boldsymbol{W}[G] .
$$

Proof. We shall use the same notation $K_{f}$ as in Theorem 10, i.e., $K_{f}$ is a set of measures such that $f \in \mathfrak{w}\left(K_{f} ; C_{0}\right) \subset F$ and $K_{f} \subset G$. By the assumption, Lemmas 1,2 and 3 and by Ascoli theorem, i) $X$ is $\mathscr{T}_{\mathfrak{w}}$-compact, ii) for any $f$ in $F, \mathfrak{t}\left(K_{f} ; \mathfrak{w}(X)\right)=\mathfrak{w}\left(K_{f} ; \mathfrak{w}(X)\right) \supset \mathfrak{w}\left(K_{f} ; C\right)=\mathfrak{w}\left(K_{f} ; C_{0}\right)$, and iii) $\mathscr{T}_{\mathfrak{w}}{ }^{X}$, or equivalently $\mathscr{T}_{\mathfrak{u}}{ }^{X}$, is weaker than $\mathscr{T}_{\mathfrak{w}}^{C_{0}}$ on $\mathfrak{w}\left(K_{f} ; C_{0}\right)$. Let $f_{1}, f_{2}, \ldots$, be a sequence of measures in $K_{f}$ such that $f_{0}(x)=\lim _{n \rightarrow \infty} f_{n}(x)$

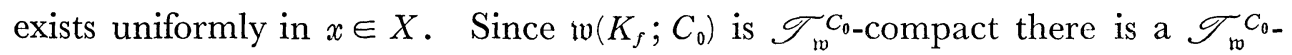
cluster point $g$ in $\mathfrak{w}\left(K_{f} ; C_{0}\right)$ of $\left\{f_{n}\right\}$, which is also a $\mathscr{T}_{\mathfrak{u}}{ }^{X}$-cluster point. Therefore $g(x)=f_{0}(x)$ for every $x \in X$, and so we have $\mathfrak{u}\left(K_{f} ; X\right)=$ $\mathfrak{w}\left(K_{f} ; C_{0}\right) \subset F$. Hence from the assumption that every element of $\mathfrak{t}\left(K_{f} ; X\right)$ admits a unique Bayes function, it follows from Theorem 7 and Lemma 1 that $\boldsymbol{B}\left[\mathfrak{w}\left(K_{f} ; C_{0}\right)\right] \subset \mathfrak{w}\left(\boldsymbol{B}\left[K_{f}\right]\right)$. Thus we observe that the Bayes function relative to $f$ belongs to $\mathfrak{w}\left(\boldsymbol{B}\left[K_{f}\right]\right)$, and consequently to $\mathfrak{w}(\boldsymbol{B}[G])$. Combining with Theorem 9, we complete the proof.

In the remainder of this section we shall denote by $P$ the set of all probability measures (positive Radon measures $f$ with $\|f\|=1$ ) and by $P_{1}$ the set of all probability measures concentrating to finite sets of points in $T$. 
Theorem 12. Suppose that $X$ is relatively $\mathscr{T}_{\mathfrak{u}}$-compact in $C$ and $\sup _{t \in T}$ $\sup _{x \in X} x(t)<\infty$. Then we have

$$
\mathfrak{w}\left(\boldsymbol{B}\left[P_{1}\right]\right) \subset \boldsymbol{W}\left[P_{1}\right] .
$$

Proof. Since $\left|\int x d f-\int y d f\right| \leqq \sup _{t \in T}|x(t)-y(t)|, \quad P_{1}$ is equicontinuous on $\left(\mathfrak{u}(X), \mathscr{T}_{\mathfrak{u}}\right)$. From Ascoli theorem it follows that $P_{1}$ is relatively $\mathscr{T}_{\mathfrak{t}} \mathfrak{u}(X)_{-}$

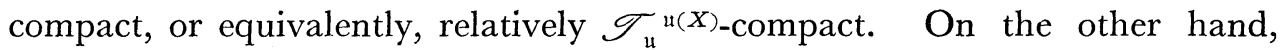
the equivalence of $\mathscr{T}_{\mathfrak{u}}$ and $\mathscr{T}_{\mathfrak{w}}$ on $X$ is implied from the $\mathscr{T}_{\mathfrak{u}}$-compactness of $\mathfrak{t}(X)$ and Ascoli theorem. Therefore by Theorem 4 we have

$$
\mathfrak{w}\left(\boldsymbol{B}\left[P_{1}\right]\right) \subset \boldsymbol{B}\left[\mathfrak{u}\left(P_{1} ; \mathfrak{t}(X)\right)\right]=\boldsymbol{W}\left[P_{1}\right] .
$$

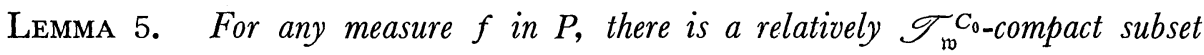
$K$ of $P_{1}$ such that

$$
f \in \mathfrak{w}\left(K ; C_{0}\right) \subset P .
$$

Proof. If $T$ is compact, our Lemma is trivial. So we shall begin with considering the case where $T$ is $\sigma$-compact. Since $T$ is locally compact, there is an ascending sequence $\left\{C_{n}\right\}$ of compact subsets of $T$ such that $\cup C_{n}=T$ and $C_{n}$ is contained in the interior of $C_{n+1}$. Let $f$ be an arbitrary measure in $P$. For any neighborhood $V$ of $f: V=V_{\mathfrak{w}}\left(f: x_{1}, x_{2}, \ldots, x_{r}, \varepsilon\right)$ with $x_{i} \in C_{0}, i=1,2, \ldots, r$, and with $\varepsilon>0$, there corresponds the smallest $C_{m}$, in the sequence $\left\{C_{n}\right\}$, containing all the supports of $x_{1}, x_{2}, \ldots$, and $x_{r}$. Since $T$ is regular ([1, Ch. 1]), for each $t \in T$ we can choose a neighborhood $U(t)$ of $t$ so that it satisfies: (i) for every $s$ in $U(t),\left|x_{i}(t)-x_{i}(s)\right|$ $<\varepsilon / 2, i=1,2, \ldots, r$, and (ii) if $t \in C_{n}-C_{n-1}$, then $U(t)$ is disjoint with $X-C_{n+1}$. Since $C_{m}$ is compact, there is a finite covering $U\left(t_{1}\right), \ldots, U\left(t_{p}\right)$ of $C_{m}$. Write $E_{1}=U\left(t_{1}\right) \cap C_{m}$ and $E_{i}=U\left(t_{i}\right) \cap C_{m}-\bigcup_{j=1}^{i-1} E_{j}, \quad i=2, \ldots, p$, and take $p+1$ points $s_{i} \in E_{i}, \quad i=1,2, \ldots, p$, and $s_{p+1} \in C_{m+1}-C_{m}$. Let $f_{V}$ be a measure in $P_{1}$, defined by $f_{V}\left(s_{i}\right)=f\left(E_{i}\right), i=1,2, \ldots, p$, and $f_{V}\left(s_{p+1}\right)=1-f\left(\bigcup_{j=1}^{p} E_{j}\right)$. It is clear that $f_{V} \in V$. Let $K$ be the set of all $f_{V}$ corresponding to neighborhoods $V$ of $f$. Thus we have $f \in \mathfrak{w}\left(K ; C_{0}\right)$. Since $f\left(C_{n}\right) \leqq f_{V}\left(C_{n}\right)$ for every $V$ and $n, \lim _{n \rightarrow \infty} f_{V}\left(C_{n}\right) \geqq \lim _{n \rightarrow \infty} f\left(C_{n}\right)=1$

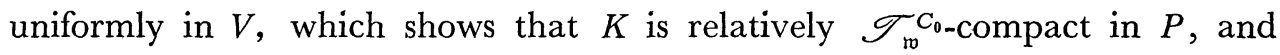
$\mathfrak{w}\left(K ; C_{0}\right) \subset P$. 
We shall proceed to the case where $f$ is not $\sigma$-compact. Since $f$ is a finite measure, there is a $\sigma$-compact and locally compact subset $T^{\prime}$ of $T$ with $f\left(T^{\prime}\right)=1$. Let $C_{0}^{\prime}, C^{\prime}$ and $K^{\prime}$ be the classes of functions and measures defined on $T^{\prime}$ correspondingly to the classes $C_{0}, C$ and $K$ on $T$ in the previous

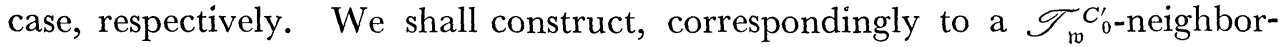
hood $V$ of $f$, a measure $f_{V}^{\prime}$, on $T$, of finite support in $T^{\prime}$ in a similar way to the $\sigma$-compact case. We shall define a measure $f_{V}$ in $P_{1}$ as $f_{V}(E)=$ $f_{V}^{\prime}\left(E \cap T^{\prime}\right)$, and denote by $K$ the set of all $f_{V}$ for $V=V_{w 0}\left(f: x_{1}^{\prime}, x_{2}^{\prime}, \ldots, x_{p}^{\prime}, \varepsilon\right)$, where $x_{1}^{\prime}, x_{2}^{\prime}, \ldots, x_{p}^{\prime}$ are in $C_{0}^{\prime}$ and $\varepsilon>0$. We can see directly that $K$ is relatively $\mathscr{T}_{\mathfrak{w}} C_{0}$-compact as a set of measures on $T$. To see $f$ being in $\mathfrak{w}\left(K ; C_{0}\right)$, we take a finite set $\left\{y_{1}, y_{2}, \ldots, y_{q}\right\}$ of elements of $C_{0}$ and a positive $\varepsilon$. Since the restrictions of these $y^{\prime}$ 's to $T^{\prime}$ are elements of $C^{\prime}$ on $T^{\prime}, K \cap V_{\mathfrak{w}}\left(f: y_{1}, y_{2}, \ldots, y_{q}, \varepsilon\right)$ can be regarded as the intersection of $K$ and a $\mathscr{T}_{\mathfrak{w}}^{C^{\prime}}$-neighborhood of $f$. Since $K^{\prime}$ is relatively $\mathscr{T}_{\mathfrak{w}}{ }^{\prime}{ }_{0}^{\prime}$-compact in $P, \mathscr{T}_{\mathfrak{w}}^{C^{\prime}}$ and $\mathscr{T}_{\mathfrak{w}}^{C^{\prime}}$ coincide with each other on $K^{\prime}$. Therefore every $K \cap V_{\mathfrak{w}}\left(f: y_{1}, \ldots, y_{q}, \varepsilon\right)$ contains a measure, whose restriction to $T^{\prime}$ is in $K^{\prime}$. Thus we have $f \in \mathfrak{w}\left(K, C_{0}\right)$.

Theorem 13. Suppose that $X$ is equicontinuous and $\sup _{t \in T} \sup _{x \in X} x(t)<\infty$. Then we have

$$
\boldsymbol{B}[P] \subset \boldsymbol{W}\left[P_{1}\right]
$$

Proof. Clear from Theorem 10 and Lemma 5.

THEOREM 14. Suppose that $T$ is compact. If for any $x \in X$ there is a $\mathscr{T}_{\mathrm{w}}$-neighborhood $V$ of $x$ such that $V$ is equicontinuous and $\sup _{t \in T} \sup _{x \in V} x(t)<\infty$, then we have

$$
\mathfrak{w}\left(\boldsymbol{B}\left[P_{1}\right]\right) \subset \boldsymbol{B}[P]=\mathfrak{w}(\boldsymbol{B}[P]) \text { and } \boldsymbol{W}\left[P_{1}\right] \subset \boldsymbol{B}[P]=\boldsymbol{W}[P] .
$$

Proof. The compactness of $T$ implies that $\mathfrak{w}\left(P_{1} ; C_{0}\right)=P$. Therefore our theorem is shown directly by Theorem 8 .

Theorem 15. If $T$ is compact, and if $X$ is equicontinuous and $\sup _{t \in T}$ $\sup _{x \in X} x(t)<\infty$, then we have

$$
\mathfrak{w}\left(\boldsymbol{B}\left[P_{1}\right]\right) \subset \boldsymbol{B}[P]=\mathfrak{w}(\boldsymbol{B}[P])=\boldsymbol{W}\left[P_{1}\right]=\boldsymbol{W}[P] .
$$


\$. Comments on Bayes class and Wald class relative to a class of additive functionals. Suppose that $F$ be a family of real functions on an arbitrary space $X$, and that $\alpha_{f}$ and $\beta_{f}$ are constant real numbers such that $\alpha_{f}>0$ and they do not depend on $x \in X$ but may depend on $f \in F$. We shall write $F^{*}=\left\{\alpha_{f} f+\beta_{f}: f \in F\right\} . \quad$ As is easily seen, we have $\boldsymbol{B}\left[F^{*}\right]=\boldsymbol{B}[F]$, but not always $\boldsymbol{W}\left[F^{*}\right]=\boldsymbol{W}[F]$. There are many important cases where $\mathfrak{w}(F)$ contains a constant function. In such a case $\boldsymbol{B}[\mathfrak{w}(F)]$ coincides with the whole space $X$, and so most relations we got in the previous two sections become useless. To avoid this unfavorableness, we should find $\alpha_{f}$ and $\beta_{f}$ so that $\mathfrak{w}\left(F^{*}\right)$ is at least $\mathscr{T}_{\mathfrak{w}}$-compact (the $\mathscr{T}_{\mathfrak{w}}$-compactness is the weakest condition among those of our theorems) and any constant function is not contained in $\mathscr{T}_{\mathfrak{w}}$-closure of $F^{*}$. This is a general principle of reducing the limit points of the Bayes class to the generalized Bayes points.

Suppose, for instance, that $F$ is a class of real additive functionals defined on a linear space $L$ containing $X$ as its subset. Let $x_{0}$ be a point of $L$ and $\phi$ an additive mapping of a linear space $M$ into $L$ such that every element $x$ of $X$ is decomposed into the sum of $\phi(y), y \in M$, and $x_{0} \in L: x=x_{0}+\phi(y)$. If $f$ is a real additive functional on $L$, then we have $f(x)=f \phi(y)+f\left(x_{0}\right)$ and $f \phi$ is an additive functional on $M$. Suppose that for any $f$ in $F$ there corresponds a positve number $\alpha_{f}$ such that $0<\inf _{f \in F} \sup _{y \in Y} \alpha_{f} \cdot f \phi(y)$ $\leqq \sup _{f \in F} \sup _{y \in Y} \alpha_{f} \cdot f \phi(y)<\infty$, where $Y=\phi^{-1}\left(X-x_{0}\right)$. Let $G=\left\{\alpha_{f} \cdot f \phi\right.$ : $f \in F\}$ and $F^{*}=\left\{\alpha_{f} f+f\left(x_{0}\right): f \in F\right\}$. Since $G$ is relatively $\mathscr{T}_{\mathfrak{w}}^{X}$-compact by Tychonoff theorem, it follows from Theorem 2 that $\boldsymbol{W}[G] \subset \boldsymbol{B}[\mathfrak{w}(G)]$, that is, $\boldsymbol{W}\left[F^{*}\right] \subset \phi(\boldsymbol{B}[\mathfrak{w}(G)])+x_{0}$. If $L$ is a topological linear space and $f \phi$ is a real continuous and additive functional on $M$ for every $f \in F$, and if $\mathfrak{n}(G ; Y)$ is $\mathscr{\mathscr { T }}_{\mathfrak{u}}^{Y}$-compact, then by Theorem $4 \mathrm{c}(\boldsymbol{B}[G]) \subset \boldsymbol{B}[\mathfrak{t}(G ; Y)]$ holds and hence $\mathfrak{c}(\boldsymbol{B}[F]) \subset \phi(\boldsymbol{B}[u(G, Y)])+x_{0}$. Since $\alpha_{f} f \phi(y)$ is an additive functional, we can easily verfy that $\mathfrak{u}(G ; Y)$ is a class of additive functionals. In [2] $\mathfrak{t}(G ; Y)$ is a class of distributions, while in [5] it is a class of $\sigma$ finite measures.

\section{REFERENCES}

[1] N. Bourbaki: Topologie générale, Actualité Sci. Ind. (1949), Paris.

[2] H. Kudō: Locally complete class of tests, Bull. Inst. Intern. Statist. 38 (1961), 173-180.

[3] T. Kusama: On classes of Bayes solutions, Ann. Inst. Statist. Math. 18 (1966), 1-11.

[4] L. LeCam: An extension of Wald's theory of statistical decision functions, Ann. Math. Statist. 26 (1955), 69-81. 
[5] J. Sacks: Generalized Bayes solutions in estimation problems, Ann. Math. Statist. 34 (1963), 751-768.

[6] A. Wald: Statistical decision functions, 1950, Wiley.

\section{Osaka City University}

